\title{
Oral sildenafil therapy for the management of mild persistent pulmonary hypertension of the newborn in term neonates
}

\author{
Poonam Singh ${ }^{1}$, Jaya Upadhyay ${ }^{2}$, Kanhu Charan Digal ${ }^{2}$, Yash Shrivastava ${ }^{3}$, Sriparna Basu $^{4}$ \\ From ${ }^{1}$ Assistant Professor, ${ }^{2}$ Resident, ${ }^{4}$ Professor and Head, Department of Neonatology, ${ }^{3}$ Assistant Professor, Department of Pediatrics, All India \\ Institute of Medical Sciences, Rishikesh, Uttarakhand, India
}

\begin{abstract}
The use of sildenafil leads to an improvement in oxygenation and has the potential to reduce mortality in severe persistent pulmonary hypertension of neonates (PPHN). Mild PPHN in term neonates often prolongs the duration of oxygen requirement without other major morbidities, thereby increasing the length of hospital stay. We present a case series of eight term neonates with clinical and echocardiographic evidence of mild PPHN, in whom treatment with oral sildenafil resulted in early clinical improvement. It was observed that neonates could be weaned off from oxygen inhalation after a mean (SD) 53.1 (11.8) h of starting oral sildenafil as their oxygen saturation was maintained between 93 and 95\% in room air. Infants could be discharged home after a median (IQR) 10 (7.5-12) days and oral sildenafil could be stopped after a median duration of $1.4(1-1.8)$ months. All infants tolerated oral sildenafil well without any major adverse events.
\end{abstract}

Key words: Neonate, Persistent pulmonary hypertension, Pulmonary vasodilator, Sildenafil

$\mathrm{P}$ ersistent pulmonary hypertension of the newborn (PPHN), characterized by a sustained elevation of pulmonary vascular resistance with right-to-left shunting, occurs in 1.8 per 1000 live births [1]. The clinical spectrum of PPHN varies from mild to severe hypoxemia requiring mechanical ventilation in $66.6 \%$ [1]. Pulmonary vasodilators, inhaled nitric oxide (iNO), and sildenafil are the mainstay of management of PPHN along with supportive therapy [2]. Although iNO is the only therapy approved by the US Food and Drug Administration for the management of PPHN in terms of near-term newborn infants [2], the majority of neonatal intensive care units (NICUs) in India use sildenafil as the first-line agent due to non-availability of iNO facility.

The cases of mild PPHN, apparently well-nourished and breastfeeding neonates, are often seen to remain admitted in NICUs only for oxygen supplementation, as weaning from oxygen results in prompt desaturation and cyanosis. Since prolonged oral doses of sildenafil have been proved to be beneficial for the management of severe PPHN associated with bronchopulmonary dysplasia (BPD) in preterm infants [3,4], we speculated that it could have a role in term neonates with mild PPHN. In this study, we present a series of eight neonates with mild PPHN admitted

\section{Access this article online}

Received - 24 January 2020

Initial Review - 15 February 2020

Accepted - 18 March 2020

DOI: 10.32677/IJCH.2020.v07.i10.005 in our NICU during the past 1 year in whom sildenafil therapy expedited weaning from oxygen resulting in early discharge.

\section{CASE REPORT}

Clinical and echocardiographic details of neonates who were diagnosed as mild PPHN and received oral sildenafil therapy are summarized in Table 1. Mild PPHN was defined by the presence of mild respiratory distress (Downes score $<4$ ), labile hypoxemia (oxygen saturation $<90 \%$ ) requiring oxygen inhalation by a hood or nasal prongs, with/without pre- and post-ductal oxygen saturation difference of $>5 \%$ along with pulmonary pressure $>30 \mathrm{mmHg}$, right-to-left/bidirectional shunt at patent ductus arteriosus (PDA) and/or patent foramen ovale (PFO), tricuspid regurgitation, and flattening of the interventricular septum by 2-D echocardiography and color Doppler, after exclusion of other congenital heart diseases.

Chest radiograph, arterial blood gas, and sepsis screen including blood culture were done to evaluate pulmonary parenchymal diseases and sepsis leading to PPHN. Oral sildenafil was started at a dose of $1 \mathrm{mg} / \mathrm{kg} /$ dose every $8 \mathrm{~h}$. If no response was observed after $24 \mathrm{~h}$, the dose was increased to $1.5 \mathrm{mg} / \mathrm{kg} / \mathrm{dose}$ every $8 \mathrm{~h}$. Other supportive management was provided as per our NICU protocol. Infants were monitored for adverse effects.

Correspondence to: Dr. Yash Shrivastava, Department of Pediatrics, All India Institute of Medical Sciences, Rishikesh - 249 203, Uttarakhand, India. E-mail: dr.yash.24@gmail.com

(C) 2020 Creative Commons Attribution-NonCommercial 4.0 International License (CC BY-NC-ND 4.0). 


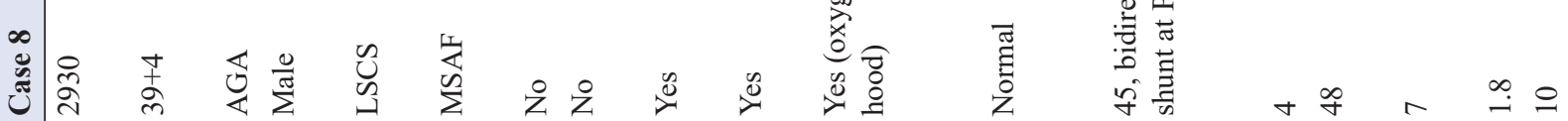

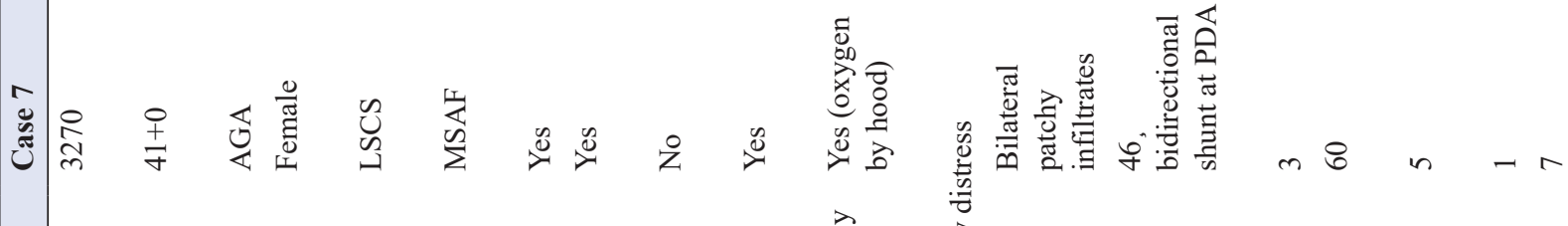

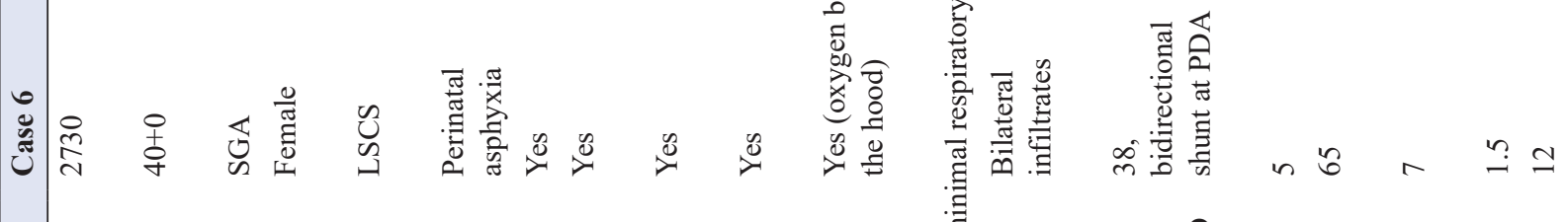

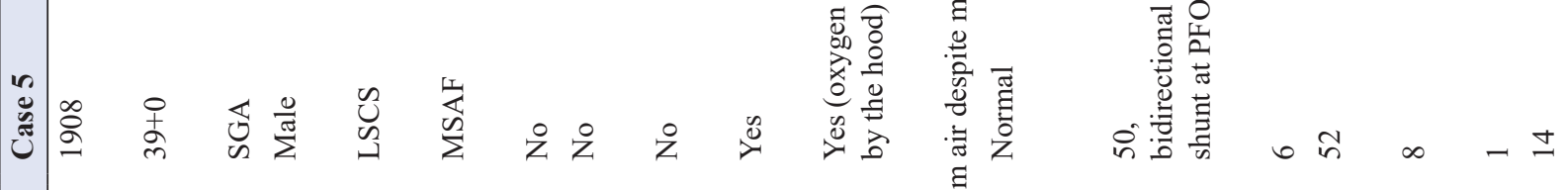

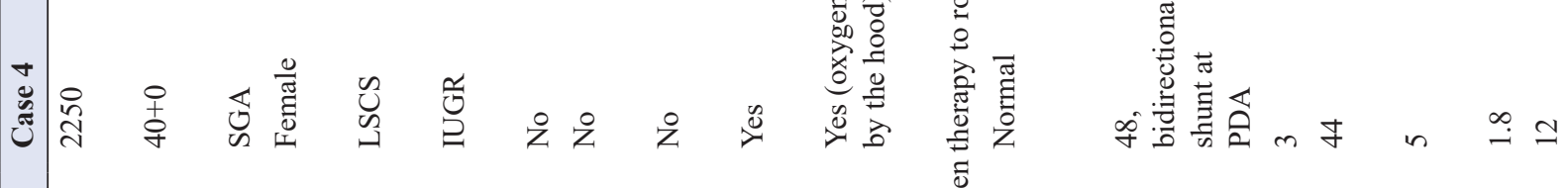

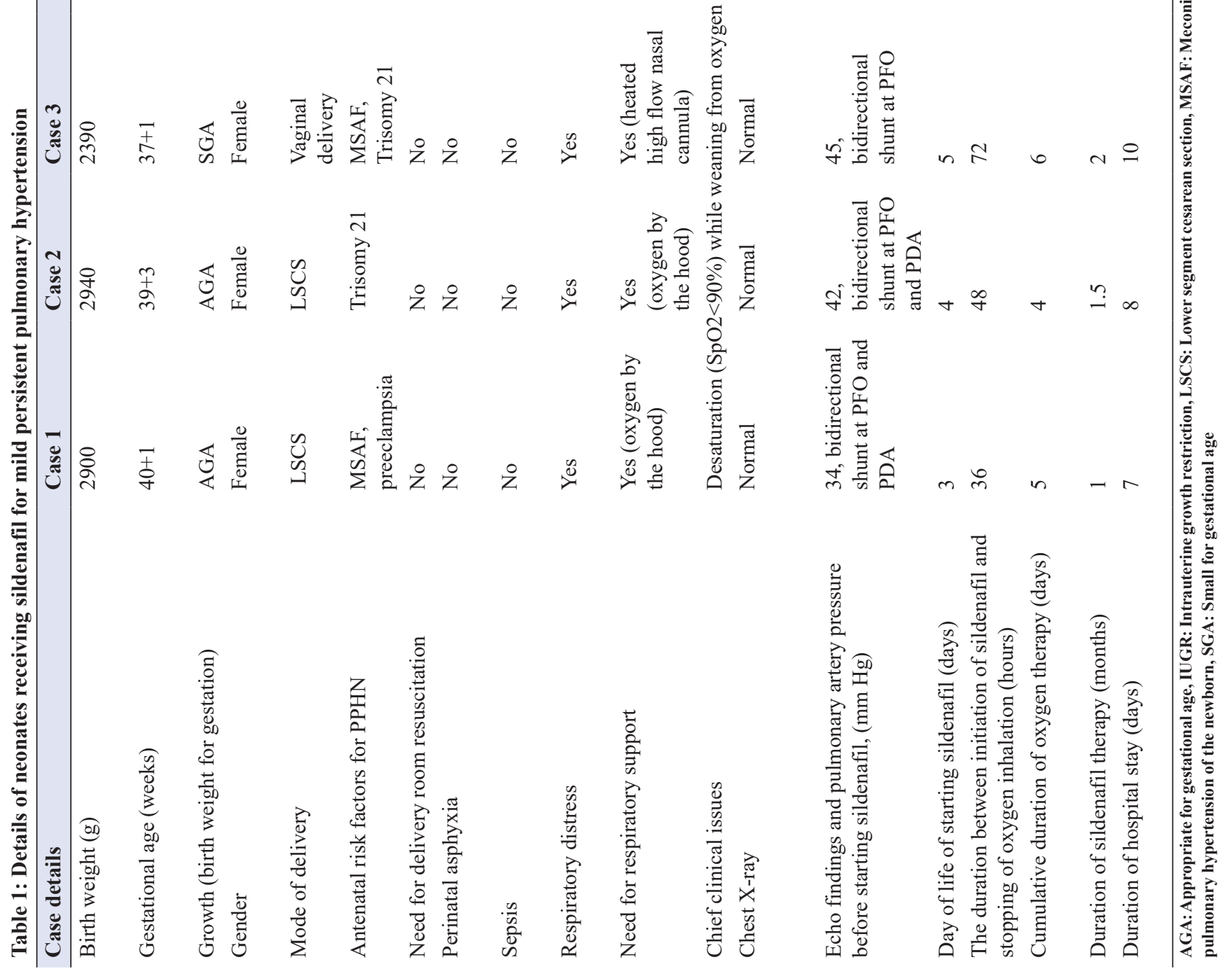


Neonates could be weaned off oxygen after a mean (standard deviation [SD]) $53.1(11.8) \mathrm{h}$ of oral sildenafil and were discharged home after a median (interquartile range [IQR]) 10 (7.5-12) days once they could maintain saturation $>90 \%$ at room air. Mean (SD) pulmonary arterial pressure to start sildenafil was 43.5 (5.3) mmHg. After discharge, infants were called weekly for clinical and echocardiographic evaluation. Oral sildenafil was stopped when pulmonary arterial pressure was reduced below $30 \mathrm{mmHg}$. In the present series, median duration of oral sildenafil therapy was $1.4(1-1.8)$ months. None of the neonates developed any major adverse events such as feeding intolerance, persistent vomiting, hypotension, or bleeding during oral sildenafil therapy.

Ethical approval for publication was taken from the Institutional Ethics Committee and informed consent was taken from the parents.

\section{DISCUSSION}

In the present case series, oral sildenafil therapy resulted in weaning from oxygen after an average duration of $53.1 \mathrm{~h}$. Literature supports the role of sildenafil in the treatment of severe PPHN; however, its role in the treatment of mild PPHN is uncertain. The previous studies have shown an improvement in the oxygenation index within $7-48 \mathrm{~h}$ of initiating sildenafil among cases with hypoxemic respiratory failure due to severe PPHN receiving $100 \% \mathrm{FiO}_{2}$ on a mechanical ventilator [5,6]. Prolonged use of sildenafil varying from 4 to 8 weeks has been shown to reduce pulmonary hypertension and myocardial stress in association with BPD $[5,6]$.

In a retrospective review of 21 infants treated with sildenafil for BPD-associated PPHN showed a reduction in median pulmonary pressure from 56.5 to $34.3 \mathrm{mmHg}$ after 4 weeks of slidenafil therapy. There was also a significant reduction in $\mathrm{FiO}_{2}$ requirement from 0.57 to 0.42 without affecting the mode of respiratory support. No serious adverse effects were noted [6].

Mourani et al. [7] reported the use of sildenafil in the management of BPD-associated PPHN in 25 children $<2$ years of age. Treatment resulted in hemodynamic improvement, defined as a $20 \%$ decrease in the ratio of pulmonary-to-systemic systolic arterial pressure or improvement in the degree of ventricular septal flattening with serial echocardiograms after a median duration of 40 days in $88 \%$ of the cases. Sildenafil was interrupted for recurrent erections in one case and intestinal pneumatosis in others.

\section{CONCLUSION}

In the present case series, oral sildenafil was found to be an effective and well-tolerated therapy in term neonates with mild PPHN to hasten weaning off from oxygen and discharge from the hospital, without any adverse effects. Larger well-designed studies may be planned to confirm the beneficial role of oral sildenafil in mild PPHN.

\section{REFERENCES}

1. Steurer MA, Jelliffe-Pawlowski LL, Baer RJ, Partridge JC, Rogers EE, Keller RL. Persistent pulmonary hypertension of the newborn in late preterm and term infants in California. Pediatrics 2017;139:e20161165.

2. Barrington KJ, Finer N, Pennaforte T, Altit G. Nitric oxide for respiratory failure in infants born at or near term. Cochrane Database Syst Rev 2017;1:CD000399

3. Kelly LE, Ohlsson A, Shah PS. Sildenafil for pulmonary hypertension in neonates. Cochrane Database Syst Rev 2017;8:CD005494.

4. Wardle AJ, Wardle R, Luyt K, Tulloh R. The utility of sildenafil in pulmonary hypertension: A focus on bronchopulmonary dysplasia. Arch Dis Child 2013;98:613-7.

5. Backes CH, Reagan PB, Smith CV, Jadcherla SR, Slaughter JL. Sildenafil treatment of infants with bronchopulmonary dysplasia-associated pulmonary hypertension. Hosp Pediatr 2016;6:27-33.

6. Tan K, Krishnamurthy MB, O'Heney JL, Paul E, Sehgal A. Sildenafil therapy in bronchopulmonary dysplasia-associated pulmonary hypertension: A retrospective study of efficacy and safety. Eur J Pediatr 2015;174:1109-15.

7. Mourani PM, Sontag MK, Ivy DD, Abman SH. Effects of long-term sildenafil treatment for pulmonary hypertension in infants with chronic lung disease. J Pediatr 2009;154:379-84.

Funding: None; Conflicts of Interest: None Stated.

How to cite this article: Singh P, Upadhyay J, Digal KC, Shrivastava Y, Basu S. Oral sildenafil therapy for the management of mild persistent pulmonary hypertension of the newborn in term neonates. Indian $\mathrm{J}$ Child Health. 2020; 7(10):415-417. 